Research Paper

\title{
Malignant Lymphomas in the Head and Neck Region - a Retrospective, Single-Center Study over 41 Years
}

\author{
Christian Walter ${ }^{1}$, Thomas Ziebart ${ }^{1}$, Keyvan Sagheb, Roman Kia Rahimi-Nedjat ${ }^{1}$, Asina Manz ${ }^{1}$, Georg \\ Hess $^{2}$ \\ 1. Oral and Maxillofacial Surgery - Plastic Surgery of the University Medical Center of the Johannes Gutenberg-University Mainz, Au- \\ gustusplatz 2, 55131 Mainz, Germany \\ 2. Department of Hematology, Oncology, and Pneumology of the University Medical Center of the Johannes Gutenberg-University Mainz, \\ Langenbeckstr. 1, 55131 Mainz, Germany
}

$\triangle$ Corresponding author: Christian Walter MD, DDS, PhD. Oral and Maxillofacial Surgery - Plastic Surgery, University Medical Center of the Johannes Gutenberg-University Mainz, Augustusplatz 2, 55131 Mainz, Germany. Phone: 0049 (0) 6131 173050; Fax: 0049 (0) 6131 176602; Email: walter@mkg.klinik.uni-mainz.de

(C) Ivyspring International Publisher. This is an open-access article distributed under the terms of the Creative Commons License (http://creativecommons.org/ licenses/by-nc-nd/3.0/). Reproduction is permitted for personal, noncommercial use, provided that the article is in whole, unmodified, and properly cited.

Received: 2014.09.04; Accepted: 2014.11.24; Published: 2015.01.07

\begin{abstract}
Objectives: Non-Hodgkin lymphomas are malignant neoplastic proliferations of the immune system that can manifest as nodal or extranodal lymphomas. The aim of this study was to retrospectively investigate the site of occurrence of lymphomas in the head and neck area and to analyze the typical symptoms of patients who presented at an oral and maxillofacial surgical department.

Material and Methods: All patient files from1971 until 2012 from an Oral and Maxillofacial Surgery of a University were analyzed for the diagnosis non-Hodgkin lymphoma. Epidemiologic data and data regarding the localization of the malignant lymphoma were evaluated.

Results: 62 patients, 34 women and 28 men with a non-Hodgkin lymphoma in the head and neck area were treated in the 41 years analyzed. In $87 \%$ of the cases the lymphoma belonged to B-cell and in $12 \%$ to the T-cell lineage. The average age at the time of diagnosis was 67 years for women $(n=34)$ and 56 years for men. With 22 patients each, the non-Hodgkin lymphoma was localized in either the soft tissues or osseous structures. In the remaining 18 cases, multiple structures were affected. In 33 patients no accompanying nodal manifestation was noticed. In 33 cases the lymphoma was located in the oral cavity. The most common symptoms were swelling (97\%), pain (40\%) and the existence of an ulcer (11\%).

Conclusion: In the present study more than $50 \%$ of the lymphomas were located in the oral cavity. Due to the unspecific symptoms, a histopathological verification of the diagnosis is crucial.
\end{abstract}

Key words: lymphoma, non-Hodgkin lymphoma, oral, head and neck

\section{Introduction}

Lymphomas are malignant neoplastic proliferations of the immune system. $10 \%$ are Hodgkin and 90\% non-Hodgkin lymphomas [1]. Up until 1990, different classifications were used making comparisons, and therefore generally accepted therapy guidelines, nearly impossible. In 1994 a new classification was implemented called REAL, standing for Revised European American Lymphoma Classification. Based on this, the current WHO classification was developed and is generally used [2].

The incidence of non-Hodgkin-lymphomas is rising in many regions and with variation in between different countries incidences increased up to $35 \%$ in the last approximately 20 years [1]. However, the survival has improved during the last decades with an increase of the 5 year survival rate of nearly $30 \%$ to 
$50.8 \%$ [1]. In the USA, 65540 new cases were diagnosed in 2007 and in the following year 20210 patients died. In the UK, 12294 new cases occurred in 2009 and 4452 died in 2010 [1]. 16230 non-Hodgkin lymphomas were diagnosed in Germany and 6003 patients died in 2010 [3].

Patients with HIV [4], organ transplantation, stem-cell transplantation, an inherited immunodeficiency syndrome, or an autoimmune disease have an increased risk to develop a non-Hodgkin lymphoma (NHL) [1] as well as patients with an increased exposure to ultraviolet radiation [5]. Microorganisms that are regularly associated with the development of a non-Hodgkin lymphoma are the Epstein-Barr virus (Burkitt's, nasal NK-cell or T-cell lymphoma), Helicobacter pylori (mucosa-associated lymphoid tissue lymphoma) [1] and HHV-8, HTLV-1, HCV, and SV40 [6].

Between 85 and $90 \%$ of all non-Hodgkin lymphomas derive from $B$ lymphocytes, and the remaining non-Hodgkin lymphomas arise from $\mathrm{T}$ lymphocytes or natural killer cells. Non-Hodgkin lymphomas usually develop in lymph nodes and most patients present with lymphadenopathy. About one third of the non-Hodgkin lymphomas are extranodal lymphomas.

Depending on the point in time and the country the study was conducted in, the proportion of lymphomas in the entire field of head and neck malignancies ranges from 1 to $17 \%[7,8]$. Surprisingly, little information is available for Western countries, and it is out of date.

Intra oral lymphomas can resemble dental abscesses [9], tumors [10] or other diseases such as osteonecrosis [11]. The knowledge of different presentation forms of non-Hodgkin lymphoma is crucial for the dentist to allow the earliest possible diagnosis and therapy for the patient.

The aim of this study was to retrospectively investigate the site of occurrence of lymphomas in the head and neck area and to analyze the typical symptoms of patients who presented at an oral and maxillofacial surgical department.

\section{Method and Materials}

All patient data files from 1971 to 2012 were reviewed for the diagnosis lymphoma. All files from the year 1971 to the year 2000 were checked manually, and the digital data files that were present as of the year 2000 were electronically searched with the search terms: lymphoma, NHL, B-cell, and T-cell.

The inclusion criterion was the diagnosis of a lymphoma. Exclusion criteria were a previously known lymphoma and a missing histological verification of the lymphoma.
Epidemiologic data were collected as well as the site of occurrence and the disease-specific symptoms described by the patient before the diagnosis was made.

The student's t-test, the Chi-Square-test and the exact Fisher's test were used for statistical analysis. A p-value $<0.05$ was considered statistically significant.

\section{Results}

In the analyzed time span from 1971 to 2012, a diagnosis of a non-Hodgkin lymphoma of the head and neck region was made for 62 patients. 52 patients $(87 \%)$ suffered from a B-cell lymphoma (28 women and 24 men (Fig. 1]), 7 patients (12\%) had a T-cell lymphoma (4 women and 3 men [Fig. 2]) and in 3 patients no data regarding the exact classification of non-Hodgkin lymphoma was available (2 women, 1 man). There was no difference in the distribution of the NHL in between the genders $(p=1.0)$.

28 patients were male $(45 \%)$ and $34(55 \%)$ were female. For all patients, the average age was 62 years $( \pm 17$ years $[y]$ standard deviation [SD]). Women $(67 \mathrm{y}$ $[ \pm 12$ y SD] $)$ were statistically significantly $(p=0.01)$ older than men 56 y ( \pm 20 y SD) (Fig. 3).

In the first analyzed decade from 1971 to 1980, 2 patients were identified; in the second decade 7 patients; in the third decade (from 1991 to 2000) 10 patients; in the fourth decade (2001 to 2010) 10 patients; and in the years 2011 and 2012 additional 5 patients were diagnosed.

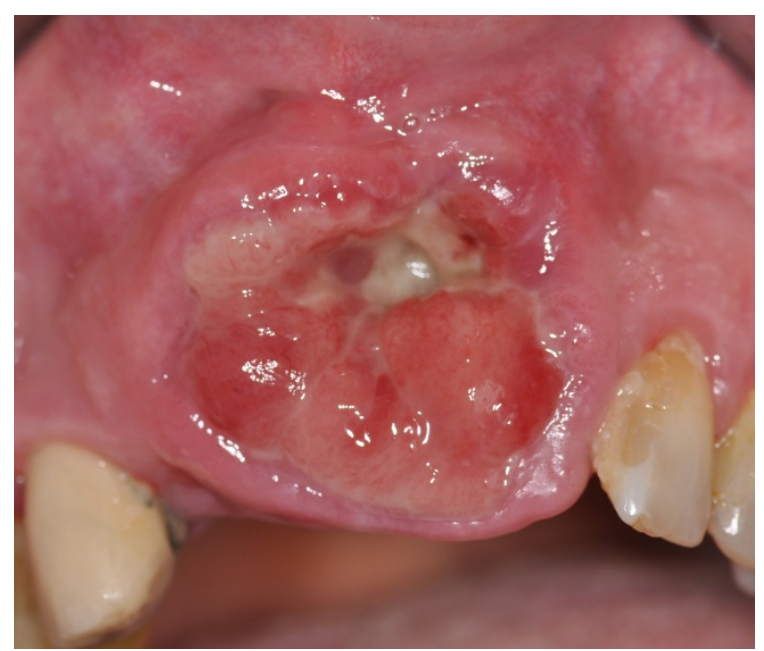

Figure 1. B-cell non-Hodgkin lymphoma. Presentation of a highly aggressive B-cell non-Hodgkin lymphoma at stage IV A. The patient's therapy was R-CHOP (cyclophosphamide, doxorubicin, vincristine, prednisolone) with an additional intrathekal triple therapy. The patient had a complete remission after 6 cycles. An additional radiotherapy of the maxilla was planned. 


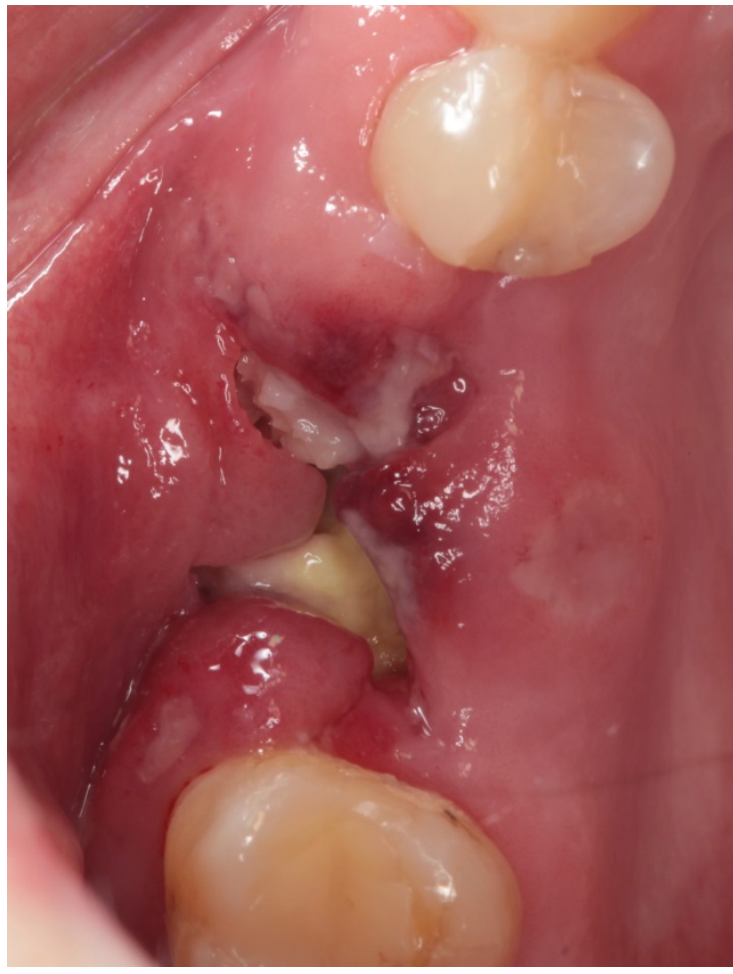

Figure 2. Mycoides fungoides. Oral manifestation of a patient with mycoides fungoides. For the cutaneous manifestations the patient had received UV A and $B$ therapy. In addition she received interferon, radiation, and chemotherapy (Gemzar and later CHOP).

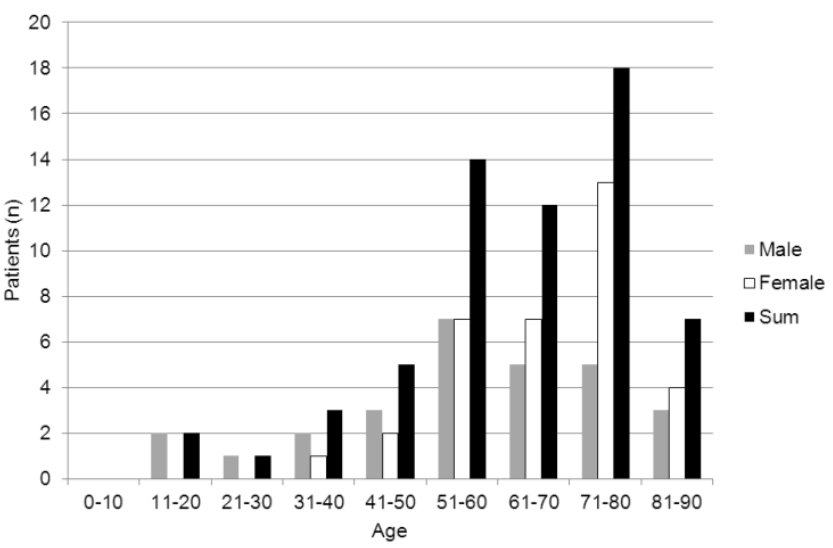

Figure 3. Age distribution. Patients with $\mathrm{NHL}$ separated by men and women. The $x$-axis describes the age groups and the $y$-axis the number $(n)$ of patients.

In 22 patients lymphoma was located in osseous structures (8 men, 14 women), and in another 22 patients the NHL occurred in the soft tissues (11 men, 11 women). In 18 patients the NHL presented at multiple sites of the head and neck ( 9 men, 9 women). Among those, 9 patients had several manifestations in the soft tissues only ( 6 men, 3 women), and in the other 9 patients osseous and soft tissues were affected ( 3 men, 9 women). In 19 patients the non-Hodgkin lymphoma presented as a nodal disease only; in 10 patients there was a combination of nodal and extranodal manifes- tation and in the remaining 33 patients no nodal manifestation was noticed (Table 1). There was no obvious pattern in the localization of the NHL in cases of several manifestations. In cases of an extranodal manifestation the potential lymph nodes were always on the same side and in some cased with additionally affected lymph nodes on the contralateral side.

Table 1: Distribution of the non-Hodgkin lymphoma manifestation sites. The first part of the table shows how many patients had manifestations at the different tissues. The second part describes the exact distribution of the different localizations since 18 patients had several spots of manifestation.

\begin{tabular}{lll}
\hline Site of occurrence & Patients / Cases (n) \\
\hline Bone & & 22 \\
Soft tissues & & 22 \\
Multiple sites & & 18 \\
Bone & Maxilla & 35 \\
& $\quad$ Mandible & 21 \\
& Periorbital region & 6 \\
Soft tissues & Calvarium & 3 \\
& & 2 \\
& Lymph nodes & 56 \\
& Salivary glands & 42 \\
& Skin & 8 \\
& Tongue & 2 \\
& Palate & 1 \\
& Temporalis muscle & 1 \\
& Mucosal membrane & 1 \\
& & 1 \\
\hline
\end{tabular}

The NHLs in osseous structures were located in the mandible in 6 cases, the maxilla in 21 cases, the periorbital region in 3 cases, and in the calvarium in 2 cases. The mandible and the maxilla were affected nearly exclusively in the posterior parts. In only 1 case each the NHL was present in the anterior region of the incisors (Fig. 1). In 2 cases each the NHL was located in the anterior and the posterior parts. The remaining NHL were located in the posterior parts.

56 NHLs were located in the soft tissues: 42 lymph nodes, 8 times in the salivary glands (parotid gland $n=5$, submandibular gland $n=2$, sublingual gland $n=1$ ), once each in the tongue, the soft palate, the temporal muscle and the buccal mucosal membrane, and twice in the skin.

The most common symptom present in 60 out of 62 patients $(97 \%)$ was a swelling, followed by pain $(n=25 ; 40 \%)$, an ulcer $(n=7 ; 11 \%)$ as well as paresthesia, redness and difficulties swallowing (each $n=4$; 6\%) (Table 2).

All patients with NHL except 7 received further treatment after diagnosis was made, such as chemotherapy or radiation therapy. In 5 patients, only surgery was performed; in one patient the disease was so advanced that no more therapy was performed, and in one patient the therapy is unknown. Follow up for 
survival was not part of this analysis, since further staging and chemo-/ radiotherapeutic treatment was not performed within the department of oral and maxillofacial surgery.

All patients with NHL except 7 received further treatment after diagnosis was made, such as chemotherapy or radiation therapy. In 5 patients, only surgery was performed; in one patient the disease was so advanced that no more therapy was performed, and in one patient the therapy is unknown. Follow up for survival was not part of this analysis, since further staging and chemo-/ radiotherapeutic treatment was not performed within the department of oral and maxillofacial surgery.

Table 2: Clinical symptoms of manifestations of non-Hodgkin lymphomas at the time of presentation.

\begin{tabular}{ll}
\hline Symptom & Occurrence in \% \\
\hline Swelling & 97 \\
Pain & 40 \\
Ulcer & 11 \\
Paresthesia & 6 \\
Redness & 6 \\
Troubles swallowing & 6 \\
\hline
\end{tabular}

\section{Discussion}

The distribution of the b-cell (87\%) and t-cell lymphomas $(12 \%)$ is in accordance with the literature [1] and so is the distribution of the age, with most patients being older than 50 years [1].

A difference was detected in the age difference between the in generally younger men compared to women, which is not a typical feature for lymphomas [3] and which is rarely described for non-Hodgkin lymphomas in the head and neck area. In addition, more than $50 \%$ of the patients did not have a nodal manifestation although a higher proportion of nodal nod-Hodgkin lymphomas is usually described in literature $[12,13]$ with a ratio of $2-3: 1$ for nodal versus extranodal manifestations. It is unknown if further manifestations were found in the following staging of these patients. It might be due to the characteristics of the patient group analyzed at an Oral and Maxillofacial Surgical Department, since the vast majority of patients are referred by dentists. Patients with a mass in the area of the neck might consult an ENT specialist instead of an oral and maxillofacial surgeon. Only few other studies have a similar distribution [14].

Usually non-Hodgkin lymphomas of the head and neck area occur more often in men, with approximately $55-77 \%$ of cases $[12,13,15,16]$. In the present study only $45 \%$ of all patients were men, so that women were slightly more often affected. This is rarely described in the literature. It might be due to the small sample size of this study's population and the missing of the exact subtype of the lymphomas [17].

$2-3 \%$ of the extranodal non-Hodgkin lymphomas appeared in the oral cavity [12]. In a recently published study about extranodal lymphomas of the head and neck region, the most common site were the salivary glands with $41 \%$. Manifestations in the mandible and the maxilla accounted for another $41 \%$, and the remaining non-Hodgkin lymphomas appeared at the paranasal sinus, the Waldeyer ring and the orbit [12]. Another recent study on 122 lymphomas in the head and neck area described 80 extranodal cases and only 42 nodal cases. Out of the 80 extranodal cases, only one appeared in the oral cavity [14].

In the present study 33 cases occurred in the oral cavity, which is more than $50 \%$. This might be due to the fact that this study was conducted in an oral and maxillofacial surgery and is therefore does not comprise all the non-Hodgkin lymphomas that have been diagnosed by the department of dermatology or the ENT.

Other limits of this study are the lack of subclassification of the non-Hodgkin lymphoma and the missing follow-up data, especially for the early patients since not all data were available anymore. On the other hand, a comparison might not be feasible, especially since the classification of lymphomas has changed several times in the past.

Approximately $5 \%$ of all malignant neoplasms of the head and neck area are malignant lymphomas [18]. The extranodal manifestation of a non-Hodgkin lymphoma, especially in the oral cavity, is thought to be a sign that the process is spreading [12]. This is not in accordance with this study's findings, since in 53\% of the patients no nodal manifestation was verified at all. Of course there could be a bias since the extranodal manifestation was the only reason the patients sought help, which might have relativized a possible concomitant lymph node manifestation that was not investigated further surgically.

A limit of this study is its retrospective design. There is always the question of correct documentation. In addition in case of nodal manifestation of the NHL in some cases most representative lymph node might have been extirpated without removing potential further ipsi- or contralateral lymph nodes.

Unfortunately the symptoms of non-Hodgkin lymphoma are not specific [15]. The most common symptom was a non-pathognomic swelling. Therefore a prompt histopathological evaluation should be sought so that early oncologic treatment can be performed as therapy is potentially curative [1] and the success depends on the kind of lymphoma [1] (Table 3 ). A delay of the diagnosis might lead to the devel- 
opment of a greater stage of lymphoma and a worse prognosis. Therefore the early detection of dental personal is of utmost importance.

This study's results are in accordance with the literature except for the large proportion of extranodal and oral manifestations and the gender distribution, with more women being affected. To analyze the differences between non-Hodgkin lymphomas manifesting at different sites of the head and neck area and between the different subtypes manifesting in this region, multicenter studies are necessary with a greater number of patients with non-Hodgkin lymphoma.

Table 3: Staging system of non-Hodgkin lymphomas according to the Ann Arbor staging system [1]. B symptoms are fever (temperature $>38^{\circ} \mathrm{C}$, night sweats, and weight loss of more than $10 \%$ of the body weight in the prior 6 months.

\begin{tabular}{|c|c|}
\hline \multicolumn{2}{|c|}{ Principal stages. Involvement of ... } \\
\hline I & ... one lymph node or one extranodal organ or site \\
\hline II & $\begin{array}{l}\text {... two or more lymph node regions on the same side of the dia- } \\
\text { phragm, or localized involvement of an extranodal site or organ } \\
\text { and one or more lymph node regions on the same side of the dia- } \\
\text { phragm }\end{array}$ \\
\hline III & $\begin{array}{l}\text {... lymph node regions on both sides of the diaphragm, potentially } \\
\text { accompanied by localized involvement of an extranodal organ or } \\
\text { site or spleen or both, (spleen accounts as nodal) }\end{array}$ \\
\hline IV & $\begin{array}{l}\text {... diffuse or disseminated of one or more distant extranodal organs } \\
\text { with or without associated involved lymph nodes }\end{array}$ \\
\hline \multicolumn{2}{|c|}{ Modifiers } \\
\hline A & Absence of B symptoms \\
\hline B & Presence of B symptoms \\
\hline
\end{tabular}

\section{Conclusion}

Typical symptoms of non-Hodgkin lymphomas manifesting in the head and neck area are swelling, pain and ulcer and it can appear as nodal and extra nodal disease. The vast majority of lymphomas does not occur in the oral cavity and therefore is rarely described. A suspicious alteration of the mucosal membrane of the oral cavity, a non-healing extraction socket or a mass at the neck should be properly diagnosed by histopathological evaluation to rule out diseases such as a malignant lymphoma and to enable early disease specific treatment.

\section{Competing Interests}

The authors have declared that no competing interest exists.

\section{References}

1. Shankland KR, Armitage JO, Hancock BW. Non-Hodgkin lymphoma. Lancet. 2012; 380: 848-57. doi:10.1016/S0140-6736(12)60605-9.

2. Swerdlow SH, Campo E, Harris NL, Jaffe ES, Pileri SA, Stein H, et al. WHO Classification of Tumours of Haematopoietic and Lymphoid Tissues. Lyon: IARC; 2008.
3. Kaatsch P, Spix C, Hentschel S, Katalinic A, Luttmann S, Stegmaier C. Robert Koch Institut Zentrum für Krebsregisterdaten - Krebs in Deutschland 2009/2010; 2013.

4. Nenasheva VV, Nikolaev AI, Martynenko AV, Kaplanskaya IB, Bodemer W, Hunsmann G, et al. Differential gene expression in HIV/SIV-associated and spontaneous lymphomas. Int J Med Sci. 2005; 2: 122-8.

5. Smedby KE, Hjalgrim H, Melbye M, Torrang A, Rostgaard K, Munksgaard L, et al. Ultraviolet radiation exposure and risk of malignant lymphomas. J Natl Cancer Inst. 2005; 97: 199-209. doi:10.1093/jnci/dji022.

6. Ekstrom-Smedby K. Epidemiology and etiology of non-Hodgkin lymphoma--a review. Acta Oncol. 2006; 45: 258-71. doi:10.1080/02841860500531682.

7. Budhy TI, Soenarto SD, Yaacob HB, Ngeow WC. Changing incidence of oral and maxillofacial tumours in East Java, Indonesia, 1987-1992. Part 2. Malignant tumours. Br J Oral Maxillofac Surg. 2001; 39: 460-4. doi:10.1054/bjom.2001.0718.

8. Parkins GE, Armah GA, Tettey Y. Orofacial tumours and tumour-like lesions in Ghana: a 6-year prospective study. Br J Oral Maxillofac Surg. 2009; 47: 550-4. doi:10.1016/j.bjoms.2008.11.003

9. Martinelli-Klay CP, Martinelli CR, Martinelli C, Dias JB, Cheade TC, Lombardi T. Primary extranodal non-Hodgkin lymphoma of the gingiva initially misdiagnosed as dental abscess. Quintessence Int. 2009; 40: 805-8.

10. Lima Mde D, Artico G, Soares FA, Martins MT, Alves FA. Follicular lymphoma in the palate with clinical appearance similar to salivary gland tumors. Quintessence Int. 2010; 41: 661-3.

11. Zadik Y, Lehman H, Neuman T, Benoliel R. Primary lymphoma of the mandible masquerading as bisphosphonate-related osteonecrosis of jaws. Quintessence Int. 2012; 43: 769-75.

12. Triantafillidou K, Dimitrakopoulos J, Iordanidis F, Gkagkalis A. Extranodal non-hodgkin lymphomas of the oral cavity and maxillofacial region: a clinical study of 58 cases and review of the literature. J Oral Maxillofac Surg. 2012; 70: 2776-85. doi:10.1016/j.joms.2012.01.018.

13. Etemad-Moghadam S, Tirgary F, Keshavarz S, Alaeddini M. Head and neck non-Hodgkin's lymphoma: a 20-year demographic study of 381 cases. Int J Oral Maxillofac Surg. 2010; 39: 869-72. doi:10.1016/j.ijom.2010.03.029.

14. Iguchi H, Wada T, Matsushita N, Oishi M, Yamane H. Anatomic distribution of hematolymphoid malignancies in the head and neck: 7 years of experience with 122 patients in a single institution. Acta Otolaryngol. 2012; 132: 1224-31. doi:10.3109/00016489.2012.694474.

15. Epstein JB, Epstein JD, Le ND, Gorsky M. Characteristics of oral and paraoral malignant lymphoma: a population-based review of 361 cases. Oral Surg Oral Med Oral Pathol Oral Radiol Endod. 2001; 92: 519-25. doi:10.1067/moe.2001.116062.

16. Mohtasham N, Babakoohi S, Sarraf-Yazdy M, Sadr B, Ghaffarzadegan K, Shiva A, et al. Oral and jaw lymphoma in an Iranian population. J Craniofac Surg. 2011; 22: 868-70. doi:10.1097/SCS.0b013e31820f7d07.

17. Salplahta D, Comanescu MV, Anghelina F, Ionita E, Mogoanta CA, Anghelina L. Non-Hodgkin lymphomas of Waldeyer's ring. Rom J Morphol Embryol. 2012; 53: 1057-60.

18. Vega F, Lin P, Medeiros LJ. Extranodal lymphomas of the head and neck. Ann Diagn Pathol. 2005; 9: 340-50. doi:10.1016/j.anndiagpath.2005.09.020. 